\title{
Iris Texture Analysis for Security Systems
}

\author{
Vanaja Roselin.E.C \\ Research scholar \\ JNTUH, Kukatpally, \\ Hyderabad-500085.
}

\author{
L.M.Waghmare, PhD. \\ Director, \\ SGGS Institute of Engineering and Technology, \\ Vishnupuri, Nanded-431602(MS)
}

\begin{abstract}
Biometric security is highly reliable and secure system. Proposed work, efficient methods for pupil detection and wavelet transformation with five-level decomposition for feature extraction are proposed and which results with accurate feature vector are stored as bits and then processes for identification and verification. Statistical performance evaluation using parameters and classifier used hamming distance for matching the patterns efficiently with stored database. We use CASIA database.
\end{abstract}

Keywords: Feature extraction, haar wavelet transformation, FAR, FRR, Feature vector size, Computational time.

\section{INTRODUCTION}

Biometrics, which refers to authentication based on his or her physiological or behavioral characteristics, its capability to distinguish authorized person and an unauthorized. Since biometric characteristics are distinctive as it cannot be forgotten or it cannot be lost, for identification person has to be present physically. Biometric is more reliable and capable than traditional knowledge based and token-based techniques. Biometric has drawback i.e., if compromised then it is difficult to replace.

Among all biometrics such as fingerprint, facial thermogram, hand geometry, face, hand thermogram, iris, retina, voice, signature etc., Iris-based identification is one of the most mature and proven technique. Iris is colored part of eye as shown in Fig1. A person's two eye iris has different iris pattern, two identical twins also has different in iris patterns because iris has many feature which distinguish one iris from other, primary visible characteristic is the trabecular meshwork, a tissue which gives the appearance of dividing the iris in a radial fashion that is permanently formed by the eighth month of gestation [17] and iris is protected by eyelid and cornea as seen in Figure1 therefore it increases security of the systems. Spoofing is very difficult with iris patterns compare to other biometrics. In practical situation it is observed that iris part is occluded by interference of eyelids and eyelashes, improper eye opening, light reflection and image quality is degraded because of low contrast image and other artifact [16]. Advantages of Iris is that it is not subject to the effects of aging which means it remains in a stable form from about age of one until death. The use of glasses or contact lenses has little effect on the representation of the iris and hence does not interfere with the recognition technology [17]. Our experiment uses wavelets such as Haar, db2, db4 for feature extraction and Hamming distance classifier used for matching process. We implement using Matlab.

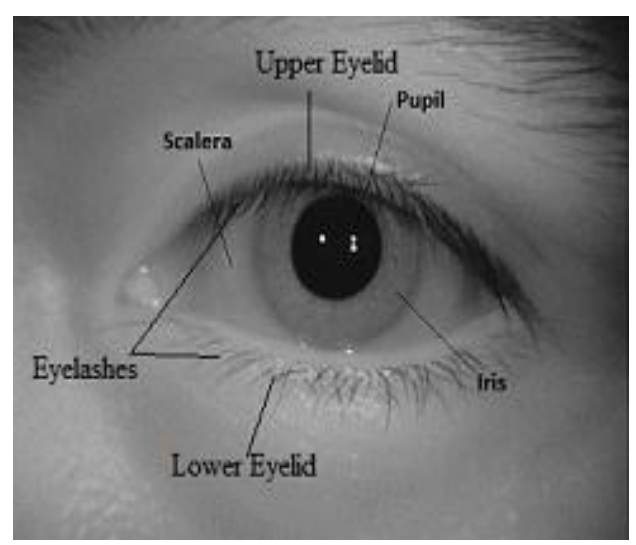

Figure1: Structure of Iris

\section{OUTLINE OF THE PAPER}

The paper is organized in the following manner; section (1) Introduction of the iris, in section (3) related work of different researcher who worked on iris recognition with feature extraction and with classifier listed in tabular form, in section (4) proposed research work with preprocessing i.e., image acquisition, iris localization \& normalization, feature extraction with section (5), section (6), section(7) and section (8).In section (9) Matching and in section (10) experimental results and discussion, finally conclusion in section (11).

\section{RELATED WORKS}

Various approaches exist in the past for iris recognition for person identification which includes John Daugman's Iriscode [1]. However proposed work uses Haar decomposition [2] for iris feature extraction to get 348-bits iris code for effective iris recognition. Advantages of haar wavelet decomposition are its computational simplicity and speed. This method is less likely to be affected by environmental factors as compared to Gabor wavelet The Iris Recognition system's main work role is to provide compact and significant feature extraction algorithm for iris images with reduced false rejection rate. The extracted feature should have high discriminating capability and the segmented iris image should be free from artifacts [13]. Daugman [2] used a multiscale quadrature twodimentional(2-D) Gabor filter to demodulate phase information of an iris image to create an Iriscode for authentication by comparing the Iriscode stored in database. Ma et al. [14] extracted features using spatial filter, this technique first converts the round image of the iris into rectangular pattern by unwrapping the circular image. Wildes et al. [9] uses Laplacian pyramid for analysis of the Iris images. Boles and Boashash [4] uses zero-crossing method with dissimilarity functions of matching. Lim et al. [3] 2D Haar Transform for feature extraction and classifier used are initialization method of the weight vectors and a new winner selection method designed for iris recognition. A. Poursaberi 
and H. N. Araabi [11] [12] use wavelet Daubechies2 for feature extraction and two classifiers such as Minimum Hamming Distance and Harmonic mean. L. Ma et al., [14] class of 1-D wavelet i.e., 1-D Intensity signals for feature extraction and for feature matching they have used expanded binary feature vector with exclusive OR operations. Md. Rabiul Islam et al., [16] used 4-level db8 wavelet transform for feature extraction and hamming distance with XOR for pattern matching. In our proposed research work we will be using wavelet family i.e., Haar wavelet, $\mathrm{db} 2$ wavelet and $\mathrm{db} 4$ wavelet for feature extraction and perform comparison on the basis of their performance evaluation. We also use Hamming Distance classifier to matching binary strings with enrolled entity in the database. To fasten the matching speed, a lower number of bits i.e., 348 bits are used in composing the iris code, as compared with other methods such as 2048 bits in [1] [2]. Comparision of iris feature extraction and classifier algorithm are as shown in Table 1.

\section{PROPOSED RESEARCH WORK}

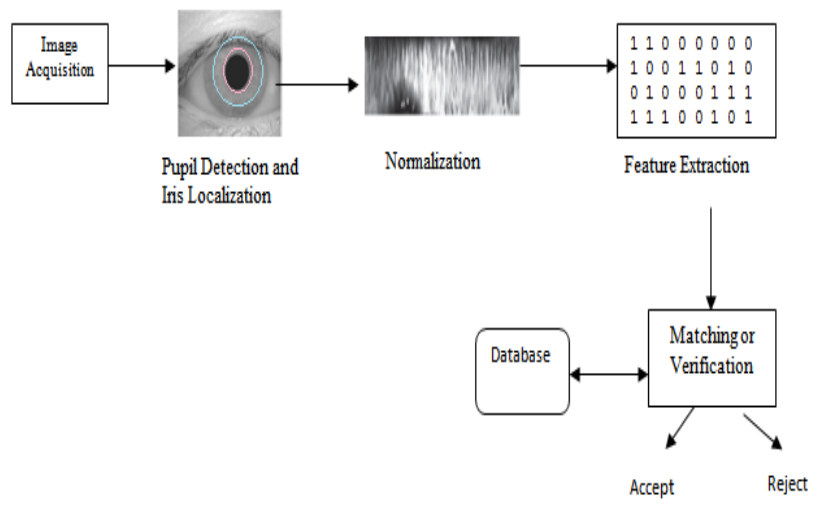

Figure2: step by step process for the proposed system

The system is consisting of 5 steps process to achieve the results. Therefore proposed systems algorithm, which is as follows:

Step 1: Image Acquisition: It is the process of acquiring image, which is done using CCD camera.

Step 2: Iris localization: when eye is captured in CCD camera, next to get only iris pattern, after extracting pupil part.

Step 3: Iris Normalization: After locating iris which is circular iris, converted to rectangular form.

Step 4: Feature Extraction: Decomposing and formation of iris pattern into iris codes.

Step 5: Matching or Verification: accept or reject by comparing stored enrolled pattern of database with submitted pattern.

\section{IMAGE ACQUISITION}

To capture high quality images for automated iris recognition systems is a major challenge. As given that the iris is a relatively small typically about 1 c.m. in diameter, and pupil is dark object, human are sensitive about their eyes, this matter requires careful engineering. Several points are of particular concern [17]. Acquiring images of Iris is major aspect of the research work with good resolution and sharpness for recognition system; need to maintain an adequate intensity of source. Acquired images must be well framed. Further, as an integral part of this process, artifacts in the acquired images (that is due to secular reflections, optical aberrations, etc.) should be eliminated as much as possible [17]. Image acquisition is considered the most critical and important step to accomplish this used a CCD camera. And set the resolution to $640 \mathrm{X} 480$, the type of the image to jpeg, and the mode to white and black for greater details. Furthermore, took the eye pictures while trying to maintain appropriate settings such as lighting and distance to camera. In this research paper we are using publicly available database i.e., Institute of Automation, Chinese Academy of science (CASIA)[29] containing 756 grayscale images of eye with 108 unique eyes or classes and seven different images of each eye are considered for our work.

\section{Iris localization}

\subsection{Pupil Detection using Scanning method}

Scanning method for pupil detection is implemented which is contribution to our research work, Daugman [7] uses Integro differential operator which has mathematical burden to system, wildes [6] uses gradient based edge detection, Poursaberi and Araabi[11] uses image morphological operator and suitable threshold. Our proposed algorithm is as follows:

Step1: Read the original image from database.

Step2: Draw Histogram of original image and calculate threshold value of pixel intensity for pupil.

Step3: Mark and fix LF as start point on $\mathrm{x}$-axis and begin scanning on $\mathrm{x}$-axis, as pupil is darker part of the eye we get dark pixel only and assign them to 0 and where we get the grey pixel that is end of the dark pixel mark and fix it as RT and assign them to 1 .

Step4: Mark and Fix UT and scan on y-axis we get dark pixel assign them to 0 and where the dark ends mark and fix it to LB assign the value as 1 .

step5: To locate center $\mathrm{C}$ of pupil compute,

$$
\mathrm{C}=\left[\left(\frac{L F+R T}{2}\right),\left(\frac{U T+L B}{2}\right)\right]
$$

Step 6: Determining pupil radius $\mathrm{PR}$

$$
\begin{aligned}
& \text { PR1 }=\text { abs }(\text { RT-C }) \\
& \text { PR2 }=\text { abs }(\text { C-LF }) \\
& \text { PR3 = abs (UB-C) } \\
& \text { PR4= abs(C-UT) }
\end{aligned}
$$

Pradius_array [PR1, PR2, PR3, PR4]

$$
\mathrm{PR}=\max \text { [Pradius_array] }
$$

Now we can locate four points on the circumference of the pupil with LF(left), RT(right), UT(UpperTop), and LB(LowerBottom) as shown in Figure6.Using region of interest based on color, we can detect the pupil but we must know the threshold value of pupil intensity. To find the threshold value of pupil intensity, draw the histogram of original image, which gives graphical representation between numbers of pixels v/s pixel intensity. As the pupil is black in color, the pupil pixel intensity lies closer to zero. Pupil has moderate size. Determine maximum number of pixels for intensity value, which is closer to zero. That value is threshold 
value of pupil intensity. If some noise occurs with pupil image, due to eyelids or eyelashes remove it. This means that there are certain pixels which lies near the pupil are of part of the iris section but having gray levels in the range of 0 to 50 . For pixels used a standard library function in the MATLAB bwareaopen( ), which removes pixels having less number of count than a certain threshold. The threshold (T) is calculated as in eq1.

$$
\left.\begin{array}{l}
g(m, n)=1 \text { if }(m, n) \leq T \\
g(m, n)=0 \text { if }(m, n) \geq T
\end{array}\right\}
$$

Figure 3 shows the original image from database, Figure 4 shows histogram of the original image, Figure 5 shows image with only pupil constructed using thresholding.

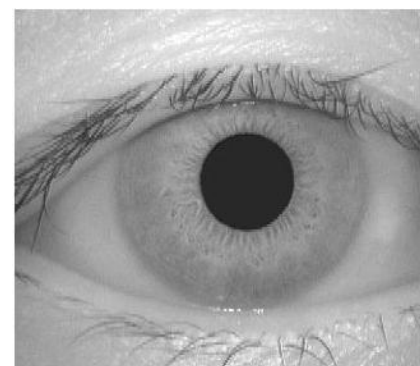

Figure 3: Original image from database

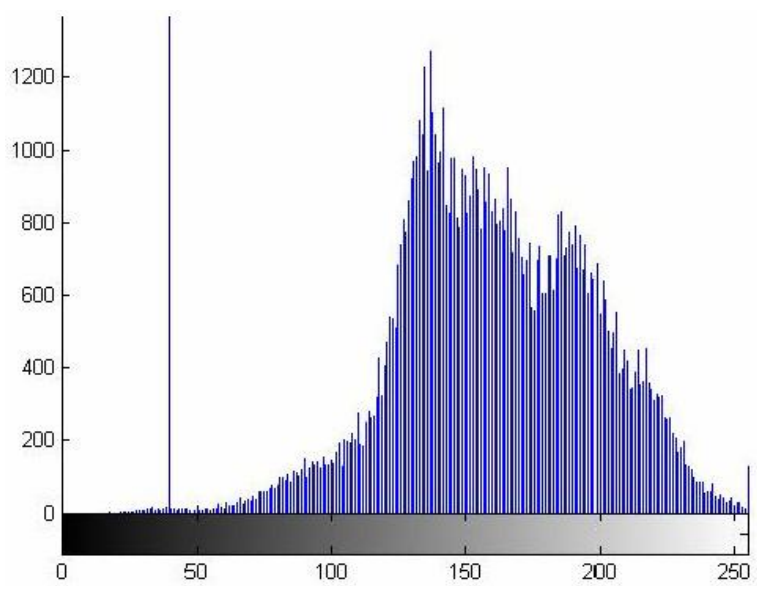

Figure 4: Histogram of original image

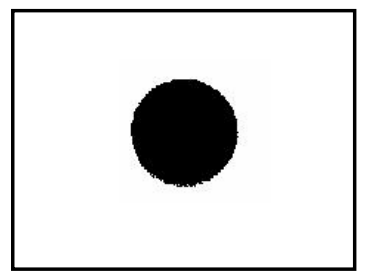

Figure 5: Image with only pupil

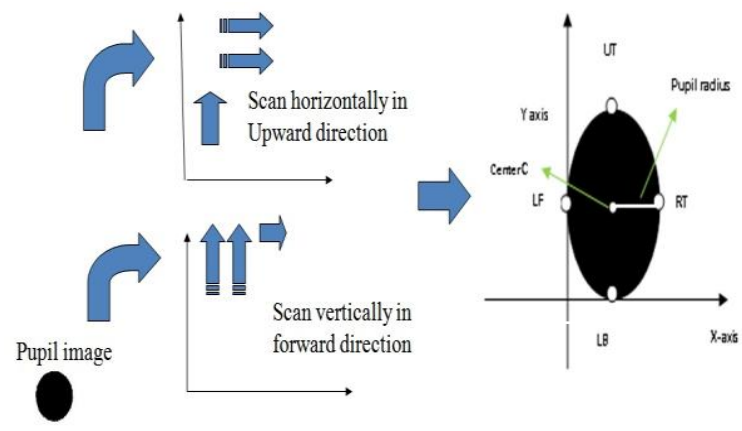

Figure 6: Four coordinate points (UpperTop(UT),

LowerBottom(LB), left(LF), and $\operatorname{right}(\mathrm{RT}))$

\subsection{Iris radius}

In our research work iris radius is calculated (as in eq2) [11]

Iris_radius $=$ pupil_radius +38

Where 38 is pixel defined in [11], add this to pupil radius to obtain Iris radius. Therefore removing iris part from total part we get major part of Iris.

\section{IRIS NORMALIZATION}

Steps for normalizing Iris image.

- Use of Daugman's rubber sheet model.

- Fixing the size of normalized figure.

- Converting normalized figure into matrix

Detection of pupil is once completed then iris section can be extracted easily. In our proposed system we consider small part of iris section for further processing so consider lower half part of iris section because most of the time upper iris section is densely covered by the eyelashes which can affect and decreases the accuracy of the system. As in our proposed work, considering CASIA database [29] which is consisting of iris images which are covered by the upper eyelashes as shown in Figure 12.Iris should be isolated and stored in a separate image because of its limits such as occlude iris part or iris covered with eye lashes and observe that possibility of pupil dilating and appearing of different size of pupil for different images. So, need to change the coordinate system by unwrapping the lower part of the iris i.e., lower 180 degree and mapping all the points within the boundary of the iris into their polar equivalent using Daugman's rubber sheet model as shown in Figure 8. The size of the mapped image is fixed which means that taking an equal amount of points at every angle. Therefore if the pupil dilates the same points will be picked up and mapped again which makes mapping process stretch invariant. Our experiment consider region of interest, which is then isolated and transformed to a dimensionless polar system. The process is achieved to be a standard form irrespective of iris size, pupil diameter or resolution. Algorithm is based on Daugman's stretched polar coordinate system. Working idea of the dimensionless polar system is to assign an $r$ and $\Theta$ value to each coordinate in the iris that will remain invariant to the possible stretching and skewing of the image. For our transformation, the $\mathrm{r}$ value ranges from $(0$ to 32 ) and angular value spans the normal ( 0 to 180 ) this means we consider only 32 pixels in each angle of $0^{\circ}, 1^{\circ}, 2^{\circ},------, 180^{\circ}$ and so on. Thus the process gives us the normalized image. 


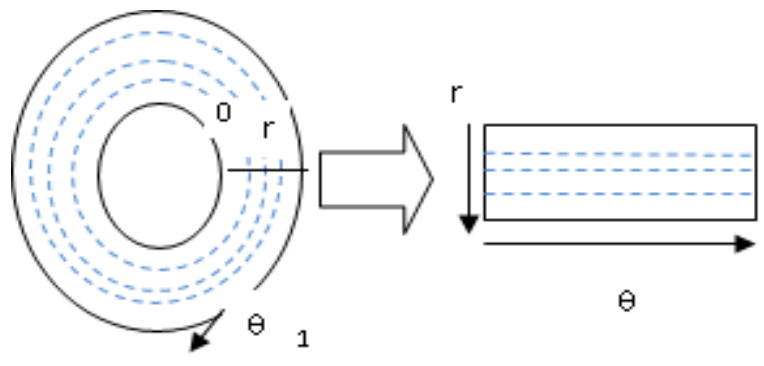

Figure 7: Daugman's Rubber sheet model with annular iris zone is stretched to a rectangular block and dashed lines are sampling circles.

Remapped image is called normalized image, which is remapped for lower 180 degrees and following figures shows the results, Figure 8 (a) shows original image Figure 8 (b) shows localized iris and Figure 8(c) and (d) shows iris normalization (isolated image for lower half). The remapping of the iris image $I(\mathrm{x}, \mathrm{y})$ from raw Cartesian coordinate to polar coordinates $I(\mathrm{r}, \theta)$ can be represented (as in eq 3$)$.

$$
I(\mathrm{x}(\mathrm{r}, \theta), \mathrm{y}(\mathrm{r}, \theta)) \longrightarrow I(\mathrm{r}, \theta)
$$

Where $r$ radius lies in the unit interval $(0,1)$ and $\theta$ is the angle between $(0,2 \pi)$.

The eq. 3 yields from eq. 4 and eq. 5 and they are

$$
\begin{array}{r}
x(r, \theta)=(1-r)^{*} x_{p}(\theta)+r^{*} x_{i}(\theta) \\
y(r, \theta)=(1-r)^{*} y_{p}(\theta)+r^{*} y_{i}(\theta)
\end{array}
$$

where $\left.\left(\mathrm{x}_{\mathrm{p}}(\theta)\right), \mathrm{y}_{\mathrm{p}}(\theta)\right)$ and $\left(\mathrm{x}_{\mathrm{i}}(\theta), \mathrm{y}_{\mathrm{i}}(\theta)\right)$ are the coordinates of pupil and iris boundary points respectively.

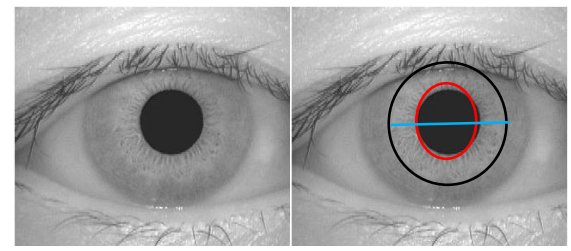

(a)

(b)

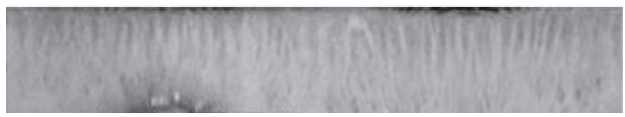

(C)

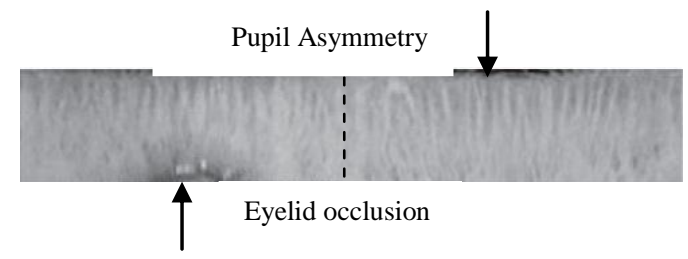

(d)

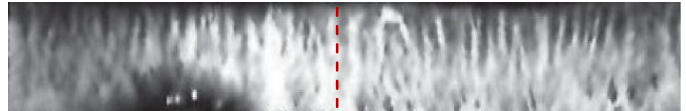

(e)

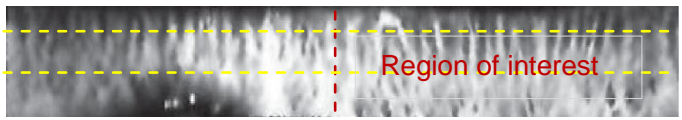

(f)

Figure 8: (a) Original Image (b) localized iris ( c) \& (d)Normalization of original image(Iris isolated image of lower half) (e) Enhanced iris (f) Region of interest

The rubber sheet model removes the deformations, hence results to $180 \mathrm{X} 32$ unwrapped sizes. Normalization not only reduces exactly distortion of the iris caused by pupil movement, but also simplifies subsequent processing.

\section{FEATURE EXTRACTION}

The iris has abundant texture information, so to provide accurate recognition of individual extract the pattern of the iris image with out noise so that quality of matching will be enhanced. In our proposed system Haar, $\mathrm{db} 2$ and $\mathrm{db} 4$ wavelet for feature extraction. The following steps for feature extraction.

1. Apply 2D DWT with Haar up to 5-level decomposition.

2. Using $4^{\text {th }}$ level, $5^{\text {th }}$ level decomposition details constructed the feature vectors.

3. Feature vectors are in the form of bianaries.

4. Store these feature vectors.

wavelet is a kind of mathematical function used to divide continous time signal into different frequency components and study each components with a resolution that matches its scales, with a scaled and translated copies of a finite-length and fast decomposed waveform is know as mother wavelet.In the research work of M. Nabti et. al.,[19] proposed the feature extraction using wavelet maxima components first and then applying Gabor filter bank to extract all features. The decomposition level considered by Shimaa M. Elserief et. al.,[22] are four level using 2D discrete wavelet transform(DWT) with four sub bands at each stage. Gabor and Wavelet transform are typically used for analyzing the persons iris patterns and extraction of features from them[2],[3],[4],[11],[14],[21]. In our proposed system we consider the five level decomposition with 2D(DWT) as in Figure 9. Why 5-level decomposition? because decomposing images with a wavelet transform yields a multi-resolution from detailed image to approximation image in each levels, considering image of size N X M (320 X 280) and decompose upto $\mathrm{K}^{\text {th }}$ level where $\mathrm{K}=1,2,3,4,5$.The quadrants(subimages) with in images as the LH,HL,HH represents detailed ie images for horizontal, vertical and diagonal orientation in the first level. The subimages LL corresponds to an approximation image that is further decomposed resulting in two level wavelet decomposition. We obtain $5^{\text {th }}$ level wavelet tree showing all detail and approximation coefficients these levels are $\mathrm{CV}_{1}$ to $\mathrm{CV}_{5}$ (vertical coefficient), $\mathrm{CH}_{1}$ to $\mathrm{CH}_{5}$ (horizontal coefficient), $\mathrm{CD}_{1}$ to $\mathrm{CD}_{5}$ ( diagonal coefficient). After $5^{\text {th }}$ level image size can become small to be useful. 


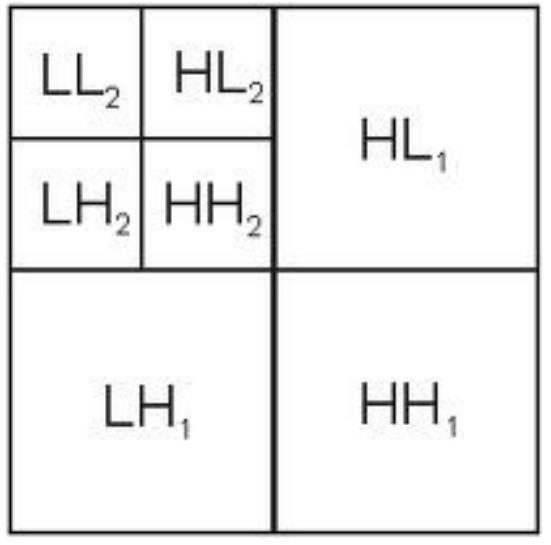

(a)

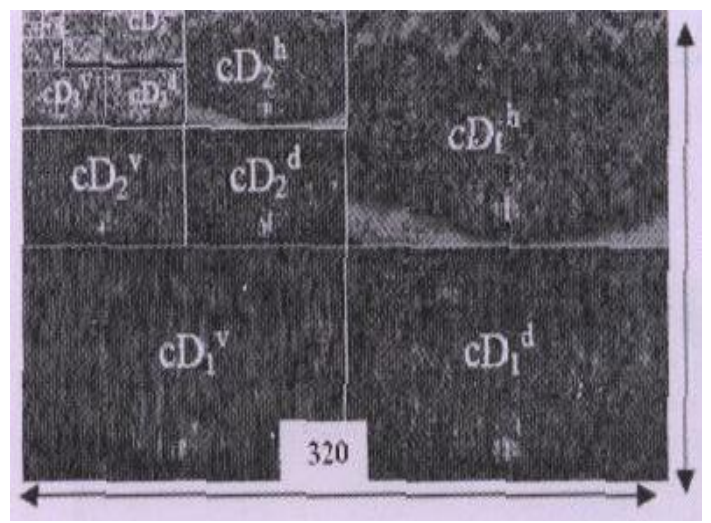

(b)

Figure 9: (a) and (b) 5- level haar wavelet decomposition

\section{MATCHING}

Calculating two irises are from the same class for their similarity Comparision between two feature vectors. Conceptualizing using Daugman's [1], [2], [7], [11], [12] we develop step by step pseudocode approach which is proposed to perform matching process using Hamming Distance.

Step 1: Compare Query image feature vector with stored image feature vector of database.

Step 2: Hamming Distance is calculated for each image feature vector.

Step 3: Finally Calculate minimum Hamming Distance.

The process of matching is identification and verification of different iris is carried out with above steps for the Comparision of two iris pattern. If Hamming Distance is greater between two feature vector than greater the difference between them. Two similar irises will fail the test since the difference between them will be small. The Hamming Distance (HD) between two Boolean vectors is defined (as in eq (6)).

$$
\mathrm{HD}=\frac{1}{N} \sum_{j=1}^{N} C_{A}(j) \oplus C_{B}(i)
$$

Where $C_{A}$ and $C_{B}$ are the coefficients of two iris images, $N$ is the size of the feature vector, Ex-OR is the Boolean operator that gives a binary 1 if the bits at the position $\mathrm{j}$ in $\mathrm{C}_{\mathrm{A}}, \mathrm{C}_{\mathrm{B}}$ are different and 0 if they are similar. Daugman [23] conducted tests on very large number of iris patterns i.e. up to 200
Billion irises images and resulted that the maximum Hamming distance that exists between two irises belonging to the same person is 0.32 .

- If $\mathrm{HD}<=$ Threshold then Match successful.

- If HD> Threshold then Match unsuccessful i.e. different person or left and right eye iris of the same person.

\section{EXPERIMENTAL RESULTS AND DISCUSSION}

\subsection{Results}

Calculate and plot Intra class distribution., testing the image with in the class and inter class testing the image with other class, we also achieve false match rate and false non match rate as seen in Figure 10, our system is giving encouraging results with false Non match rate is $0.25 \%$ and False match rate is $0.11 \%$ for Haar wavelet with different hamming distance. Fig11 signifies the score distribution for imposter and genuine for different hamming distance, it states that as if HD is less FAR reduces and FRR increases and if HD increases FAR increases and FRR decreases this leads to plot ROC curve which is as shown in Figure 12.

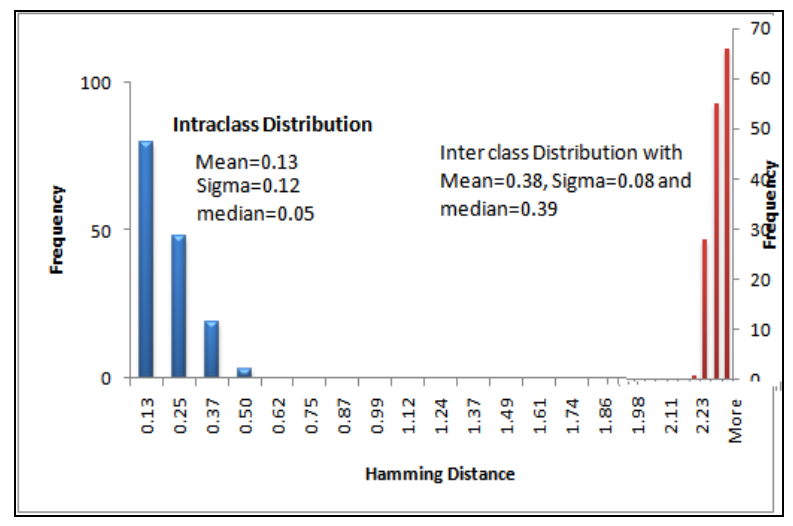

Fig10: frequency distribution of HD for intraclass and interclass

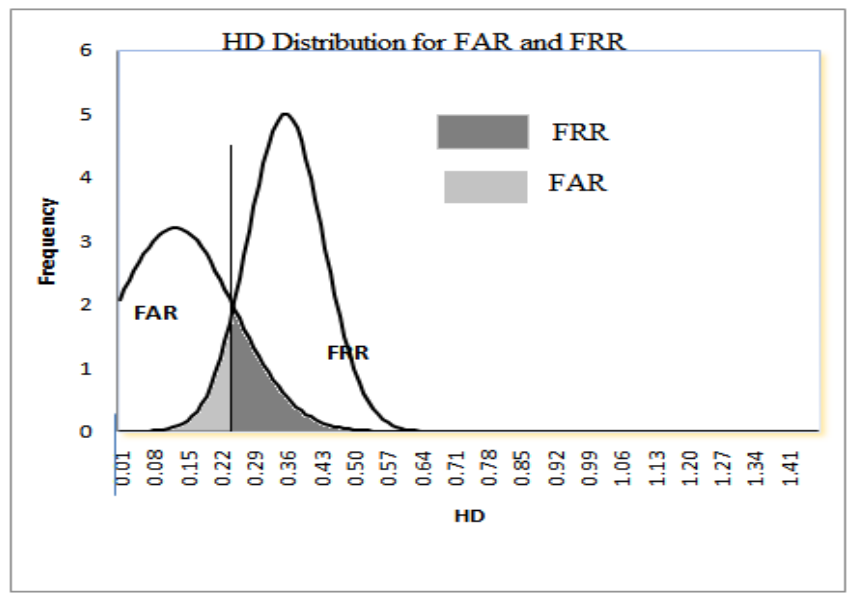

Fig11: score distribution for imposter and genuine for different hamming distance 


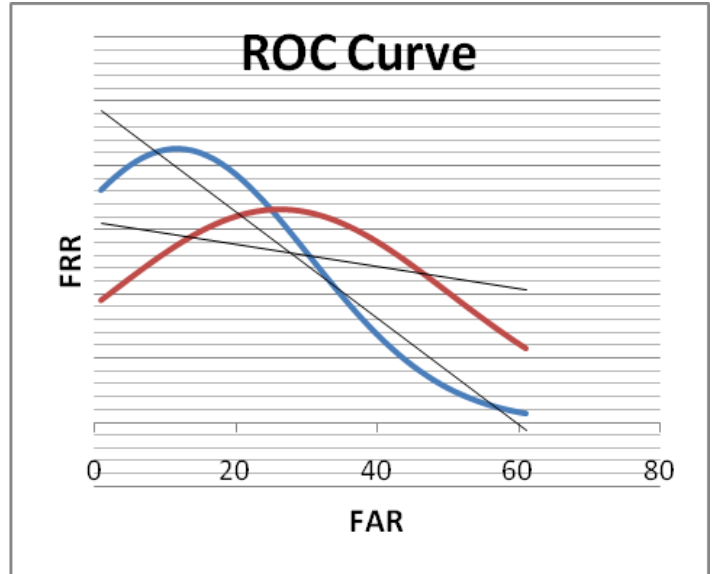

Figure 12: ROC curve for the system for different HD

\subsection{Comparision and Discussion}

The previous existing proposed methods for iris recognition by Daugman [1-2], Wildes [7], Boles et al. [5], Li Ma et al. [3] are the best know. Moreover they explain and present different way of details for iris recognition in identification and verification modes. Poursaberi [12] works on wavelet for partially occluded iris texture image, $\mathrm{Li} \mathrm{Ma}[3,15]$ also works on iris texture analysis and give encouraging results as comparing other methods Daugman results are quite encouraging in terms of accuracy and efficiency. Therefore, we analyze and compare our proposed work with exiting methods. Our method is using CASIA Iris database for verification and identification modes and found that our results are also encouraging in terms of accuracy, efficiency and reduced computational complexity. We make comparison of our results with methods $[1-2,7,3,15,12]$ of published results. Table 1 and Figure 13 give the comparison in terms of CRR and EER.

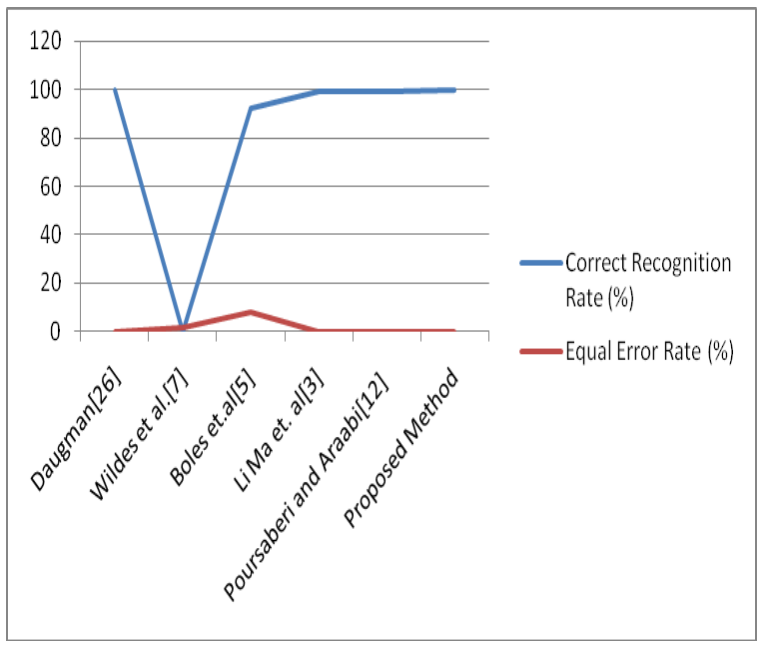

Figure 13: Comparision of CRR and EER
Table1. Comparision of CRR and EER

\begin{tabular}{ccc}
\hline Methods & $\begin{array}{l}\text { Correct Recognition } \\
\text { Rate (\%) }\end{array}$ & $\begin{array}{l}\text { Equal Error } \\
\text { Rate (\%) }\end{array}$ \\
\hline Daugman[26] & 100 & 0.08 \\
Wildes et al.[7] & - & 1.76 \\
Boles et.al[5] & 92.64 & 8.13 \\
Li Ma et. al[3] & 99.60 & 0.29 \\
Poursaberi and & 99.31 & 0.2687 \\
Araabi[12] & & \\
Proposed Method & 99.82 & 0.18 \\
\hline
\end{tabular}

\subsection{Future work}

Our experimental results demonstrates that enhance method for pupil extraction and five level decomposition for iris image has significantly encouraging and promising results in terms of EER and CRR. Our Feature work will include:

- Improving effectiveness in matching in terms of computational cost time.

- We are also currently working on global textural analysis with more levels of decomposition with accurate feature

- Extraction for larger database similar to Daugman's methods.

\section{CONCLUSION}

In this paper, enhancing iris recognition algorithm based on Haar wavelet with quality texture features of iris within feature vector, even though obstruction of eyelashes and eyelids and our proposed method also works perfect for narrowed eyelid as proposed method consider small part of the iris even though it is occluded. So, it increases the overall accuracy of the system with less computational cost in terms of time as compared with methods of Daugman[26] and Li $\mathrm{Ma}[3]$ and high recognition rate with reduced EER,FAR,FRR. The results also show the performance evaluation with different parameters with different class of variations i.e., Inter class hamming distance variation and Intra class hamming distance variation.

\section{REFERENCES}

[1] J.Daugman," How Iris Recognition works", proceedings of 2002 International conference on Image processing, vol.1, 2002.

[2] J.Daugman," High confidence visual recognition of persons by a Test of statistical Independence", IEEE Transactions on pattern analysis and machine intelligence, vol.15, no.11, Nov 1993, pp1148-1161.

[3] Li. Ma, et al., "Local Intensity Variation Analysis for Iris Recognition", Elsevier- Pattern Recognition, vol.37, No.6, pp.1287-1298, 2004.

[4] S. Lim, K. Lee, O. Byeon, T. Kim, "Efficient Iris Recognition through Improvement of Feature Vector and Classifier", ETRI Journal, Vol. 23, No. 2, June 2001, pp. 61-70.

[5] W.Boles and B.Boashash,"A Human Identification Technique using Images of the Iris and wavelet 
transform”, IEEE Transactions on signal processing, Vol. 46, No. 4, April 1998.

[6] Karu K, Anil Jain K, and Bolle R M," Is there any texture in the Image?", IEEE Transactions on Pattern Recognition, Vol. 29, No. 9, pp.1437-1446, 1996.

[7] R.Wildes et al., "Machine Vision System for Iris Recognition", Machine Vision and Applications, Vol.9, pp. 1-8, 1996.

[8] R.P.wildes," Iris Recognition: An Emerging Biometric Technology", Proceeding of the IEEE, vol. 85, pp 13481363, 1999.

[9] J.Daugman," How Iris Recognition works", IEEE Transactions on Circuits and systems for video Technology, Vol. 14, No. 1, Jan 2004.

[10] Richard Yew Fatt Ng, Yong Hour Tay, Kai Ming Mok," A Review if Iris Recognition Algorithm", IEEE Transaction, DOI 978-1-4244-2328-6/08, 2007.

[11] R.Wildes, J.Asmuth, G. Green,S. Hsu, R. Kolczynski, J. Matey and S.McBride,"A machine-vision system for Iris Recognition", Machine vision and Applications, vol.9, pp1-8,1996.

[12] Robert W. Ives, Anthony J. Guidry and Delores M Etter, "Iris Recognition using Histogram Analysis", IEEE conference on signal, system and computer ,2004, pp. $562-566$.

[13] A. Poursaberi and H. N. Araabi, "Iris Recognition for Partially Occluded Methodology and Sensitivity Analysis", EURASIP journal on "Advances in Signal processing, Vol. 2007, Article ID 36751, 2005.

[14] A.Poursaberi and B.N.Araabi, "A Half Eye Wavelet Based for Iris Recognition" proceeding of the 2005,5th International Conference on Intelligent Systems Design and Applications (ISDA'05).

[15] Amol D. Rahulkar, R.S. Holambe, "Half-Iris Feature Extraction and Recognition using a New Class of Biorthogonal triplet Half-Band Filter Bank and Flexible k-out-of-n: A postclassifier", IEEE Transactions on Information Forensics and Security, Vol. 7, No.1, Feb 2012.

[16] Li Ma, T. Yunhong Wang, and D. Zhang. "Personal identification based on iris texture analysis", IEEE Transactions on Pattern Analysis and machine Intelligence, vol.25, no.12, 2003.

[17] S. Mallat, W. Hwang, "singularity detection and processing with wavelet", IEEE Transaction on information Theory, 38(2):617-643, 1992.
[18] Md. Rabiul Islam, Dr. Wang Yin Chai, Asma Khatun," Partial Iris Image Recognition Using Wavelet Based Texture Features", International Conference on Intelligent and Advance Systems (ICIAS) ,2010.

[19] Jafar M. H. Ali, Aboul Ella Hassanien," An Iris Recognition System to Enhance E-security Environment Based on Wavelet Theory", AMO-Advance Modeling and Optimization, vol. 5, No. 2, 2003.

[20] Mayank vatsa, Richa singh, Afzel Noore," Improving Iris recognition performance using segmentation, Quality Enhancement, Match Score Fusion, and Indexing”, IEEE Transactions on Systems, Man, and Cybernetics B, Cybern., vol. 38, no. 4, pp.1021-1034, Aug. 2008.

[21] Makram Nabti, Lahouari Ghouti and Ahmed Bouridane," An effective and fast iris recognition system based on a combined multiscale feature extraction technique", Elsevier-Pattern Recognition 41, (2008) 868-879.

[22] Kevin W.Bowyer, Karen Hollingsworth, Patrick J. Flynn," Image understanding for iris biometrics: A survey", Elsevier computer vision and Image Understanding 110(2008) 281-307.

[23] Li Ma, Tieniu Tan, Yunhong Wang and Dexin Zhang, "Efficient Iris Recognition by Characterizing Key Local Variations", IEEE Transactions on Image processing, Vol. 13, No.6, June 2004.

[24] Shimaa M.Elsherief, Mahmoud E.Allam, Mohamed W. Fakhr, "Biometric Personal identification Based on Iris Recogition", International conference on computer Engineering and Systems, on page 208-213, 5-7 Nov, 2006.

[25] John Daugman, "Probing the Uniqueness and Randomness of IrisCodes: Results from 200 Billion Iris Pair Comparisons", Proceedings of the IEEE, Vol. 94, Issue: 11, pages: 1927-1935, Nov 2006.

[26] L.Ma, Y.wang, T.Tan," Iris recognition Based on multichannel Gabor Filtering", Proc. of the $5^{\text {th }}$ Asian conference on computer vision, vol.I, pp.279-283, 2002.

[27] J.Daugman, "Statistical Richness of Visual Phase Information: Update on recognizing persons by Iris patterns", International Journal of computer vision, Vol.45, No.1, pp.25-38,2001.

[28] L.Ma, Y.wang, T.Tan," Iris Recognition using Circular Symmetric Filters", proc. of the $16^{\text {th }}$ International Conference on pattern recognition, vol.II, pp.414417,2002 .

[29] http://www.sinobiometrics.com/resources.htm. 
Table1: List of iris feature extraction and Matching Algorithm of different researcher's.

\begin{tabular}{|c|c|c|c|c|c|}
\hline $\begin{array}{l}\text { Sl. } \\
\text { No. }\end{array}$ & $\begin{array}{l}\text { Researcher's } \\
\text { methods }\end{array}$ & $\begin{array}{l}\text { Feature } \\
\text { Extraction }\end{array}$ & $\begin{array}{l}\text { Matching } \\
\text { Process }\end{array}$ & $\begin{array}{l}\text { Feature } \\
\text { vector }\end{array}$ & Results \\
\hline 1 & Daugman[2] & 2D Gabor & $\begin{array}{l}\text { Hamming } \\
\text { Distance with } \\
\text { XOR }\end{array}$ & $\begin{array}{l}\text { Binary i.e., } \\
2048 \text { bit phase } \\
\text { vector }\end{array}$ & $\begin{array}{l}300 \mathrm{MHZ} \text { CPU, search } \\
\text { are performed at the } \\
\text { rate of about } 100,000 \\
\text { iris per second. }\end{array}$ \\
\hline 2 & Wildes [6] & $\begin{array}{l}\text { Laplacian } \\
\text { pyramid \& } \\
\text { Gaussian Filters }\end{array}$ & $\begin{array}{l}\text { Normalized } \\
\text { Hamming } \\
\text { Distance with } \\
\text { exclusive OR } \\
\text { operator }\end{array}$ & 256 bytes & - \\
\hline 3 & $\begin{array}{l}\text { A.Poursaberi \& } \\
\text { H.N. } \\
\text { Araabi[11][12] }\end{array}$ & $\begin{array}{l}\text { Wavelet Based } \\
\text { Feature } \\
\text { extraction }\end{array}$ & $\begin{array}{l}\text { Minimum } \\
\text { Hamming } \\
\text { Distance(MHD) } \\
\text { \& Harmonic } \\
\text { mean }\end{array}$ & $\begin{array}{l}408(544) \\
\text { binary feature } \\
\text { vector }\end{array}$ & $\begin{array}{l}\text { CRR is } 99.31 \% \text { \& } \\
\text { ERR is } 0.2687 \%\end{array}$ \\
\hline 4 & Vatsa et al.,[18] & $\begin{array}{l}\text { 1-D log polar } \\
\text { Gabor Transform } \\
\& \text { Topological } \\
\text { feature extraction } \\
\text { using Euler No. }\end{array}$ & $\begin{array}{l}2 \mathrm{v}-\mathrm{SVM} \text { method } \\
\text { for matching the } \\
\text { texture \& } \\
\text { topological } \\
\text { features }\end{array}$ & - & $\begin{array}{l}\text { Performance in terms } \\
\text { of accuracy is } 97.21 \%\end{array}$ \\
\hline 5 & $\begin{array}{l}\text { Makram Nabti } \\
\text { et al.,[19] }\end{array}$ & $\begin{array}{l}\text { Wavelet maxima } \\
\text { component as } \\
\text { multiresolution } \\
\text { technique \& } \\
\text { special Gabor } \\
\text { filter bank }\end{array}$ & $\begin{array}{l}\text { Hamming } \\
\text { Distance with } \\
\text { XOR }\end{array}$ & $\begin{array}{l}\text { Statistical } \\
\text { feature with } \\
480 \text { vector } \\
\text { elements \& } \\
\text { moments } \\
\text { invariants using } \\
1680 \text { vector } \\
\text { elements }\end{array}$ & $\begin{array}{l}\text { Feature extraction } \\
\text { computational } \\
\text { complexity (ms), } \\
\text { statistical feature: } 74 \\
\text { Moment invariants: } 81\end{array}$ \\
\hline 6. & $\begin{array}{l}\text { Amol D. } \\
\text { Rahulkar } \\
\text { et al.,[13] }\end{array}$ & $\begin{array}{l}\text { Biorthogonal } \\
\text { Triplet Half } \\
\text { Band Filter } \\
\text { Bank(THFB) }\end{array}$ & $\begin{array}{l}\text { Flexible k-out- } \\
\text { of-n: } \\
\text { postclassifier }\end{array}$ & $\begin{array}{l}7 \text { integer values } \\
\text { per region }\end{array}$ & $\begin{array}{l}\text { Low computational } \\
\text { complexity with } \\
\text { significant reduced } \\
\text { FRR. }\end{array}$ \\
\hline 7 & Lim et al.,[3] & $\begin{array}{l}\text { Haar wavelet } \\
\text { Transform }\end{array}$ & $\begin{array}{l}\text { LVQ neural } \\
\text { network }\end{array}$ & $\begin{array}{l}87 \\
\text { dimensions(1bi } \\
\text { t/dimension) } \\
\text { i.e.,87bits }\end{array}$ & $\begin{array}{l}\text { Recognition } \\
\text { performance is } 98.4 \%\end{array}$ \\
\hline 8 & L. Ma et al.,[14] & $\begin{array}{l}\text { Class of 1-D } \\
\text { Wavelets i.e., 1- } \\
\text { D Intensity } \\
\text { signals }\end{array}$ & $\begin{array}{l}\text { Expanded binary } \\
\text { Feature vector \& } \\
\text { Exclusive OR } \\
\text { operations }\end{array}$ & $\begin{array}{l}\text { Vector consists } \\
\text { of } 660 \\
\text { components \& } \\
\text { represented in } \\
\text { byte. }\end{array}$ & $\begin{array}{l}\text { CRR is } 100 \% \text { \& EER } \\
\text { is } 0.07 \% \text { \& } \\
\text { computational } \\
\text { complexity is } \\
250.7(\mathrm{~ms})\end{array}$ \\
\hline 9 & $\begin{array}{l}\text { Md. Rabiul Islam } \\
\text { et al.,[16] }\end{array}$ & $\begin{array}{l}\text { 4-level db8 } \\
\text { wavelet } \\
\text { transform }\end{array}$ & $\begin{array}{l}\text { Hamming } \\
\text { Distance with } \\
\text { XOR }\end{array}$ & $\begin{array}{l}\text { Binary codes of } \\
510 \text { bits }\end{array}$ & $\begin{array}{l}\text { CRR is } 98.14 \% \text { \& } \\
\text { ERR is } 0.21 \%\end{array}$ \\
\hline 10 & $\begin{array}{l}\text { Proposed } \\
\text { Method }\end{array}$ & $\begin{array}{l}\text { 5-level Wavelet } \\
\text { transformation } \\
\text { method such as } \\
\text { Haar,db2,db4 }\end{array}$ & $\begin{array}{l}\text { Hamming } \\
\text { Distance with } \\
\text { XOR }\end{array}$ & FV of 90 bits & $\begin{array}{l}\text { EER }=0.18 \% \\
C R R=99.82 \%\end{array}$ \\
\hline
\end{tabular}

